\title{
Current concepts in F18 FDG PET/CT-based radiation therapy planning for lung cancer
}

\author{
Percy Lee ${ }^{1}$ *, Patrick Kupelian' ${ }^{1}$, Johannes Czernin' ${ }^{2}$ and Partha Ghosh ${ }^{3}$ \\ ' Department of Radiation Oncology, David Geffen School of Medicine at UCLA, Los Angeles, CA, USA \\ ${ }^{2}$ Division of Nuclear Medicine, Department of Pharmacology, David Geffen School of Medicine at UCLA, Los Angeles, CA, USA \\ ${ }^{3}$ Siemens Healthcare, Molecular Imaging, Hoffman Estates, IL, USA
}

\section{Edited by:}

Ilja F. Ciernik, Städtisches Klinikum

Dessau, Germany

\section{Reviewed by:}

Jesper Thygesen, Region Midtjylland, Denmark

Chaitanya Divgi, Columbia University, USA

\section{${ }^{*}$ Correspondence:}

Percy Lee, Department of Radiation Oncology, David Geffen School of Medicine at UCLA, 200 UCLA Medical Plaza, B265, Los Angeles, CA, USA. e-mail:percylee@mednet.ucla.edu
Radiation therapy is an important component of cancer therapy for early stage as well as locally advanced lung cancer. The use of F18 FDG PET/CT has come to the forefront of lung cancer staging and overall treatment decision-making. FDG PET/CT parameters such as standard uptake value and metabolic tumor volume provide important prognostic and predictive information in lung cancer. Importantly, FDG PET/CT for radiation planning has added biological information in defining the gross tumor volume as well as involved nodal disease. For example, accurate target delineation between tumor and atelectasis is facilitated by utilizing PET and CT imaging. Furthermore, there has been meaningful progress in incorporating metabolic information from FDG PET/CT imaging in radiation treatment planning strategies such as radiation dose escalation based on standard uptake value thresholds as well as using respiratory-gated PET and CT planning for improved target delineation of moving targets. In addition, PET/CT-based follow-up after radiation therapy has provided the possibility of early detection of local as well as distant recurrences after treatment. More research is needed to incorporate other biomarkers such as proliferative and hypoxia biomarkers in PET as well as integrating metabolic information in adaptive, patient-centered, tailored radiation therapy.

Keywords: image-guided radiation therapy, intensity-modulated radiation therapy, lung cancer, PET/CT, radiotherapy planning, stereotactic body radiation therapy

\section{INTRODUCTION}

Lung cancer is the leading cause of cancer death. In 2007, the annual incidence was approximately 20,000 and almost 16,000 people died from lung cancer (http://www.cdc.gov/Features/ dsLungCancer). The overall 5-year survival of patients diagnosed with lung cancer is approximately $14 \%$. Only one-third of presenting patients are eligible for surgery (Rubin, 2001).

Although surgery is the therapy of choice in early-stage lung cancer, radiation therapy plays a major role in patients who are either medically or technically inoperable and not candidates for surgery. Recent advances in radiation therapy for non-small cell lung cancer (NSCLC) including intensity-modulated radiotherapy (IMRT), image-guided radiotherapy (IGRT), and stereotactic body radiotherapy (SBRT) have enabled higher radiation doses to be delivered to tumors which increases the tumor local control probability while reducing doses to surrounding normal tissue. This has led to the ability to reduce radiation-induced shortterm and long-term toxicities. Although CT-based planning is the standard approach, CT-based planning only provides anatomical information. Incorporating PET into radiation treatment planning adds a layer of biological information, glucose consumption. Improved targeting of viable tumor based on delineation of metabolically active tumor by F18 FDG PET/CT has been the basis of increased adoption of PET-based radiation treatment planning.

Intensity-modulated radiation therapy uses non-uniform radiation beam intensities in order to improve conformal target coverage especially for irregularly shaped tumors. This allows for dose escalation along with better sparing of surrounding normal tissues leading to improved treatment outcomes and decreased radiation-induced toxicity (Teh et al., 1999). IGRT incorporates planar and volumetric imaging information (X-ray, CT, and Cone-Beam CT-based) to improve the accuracy of daily patient positioning with the possibility of adaptation and modification of the target volume when the anatomy of the tumor and or normal organs changes during therapy (Wurm et al., 2006). Adaptive radiotherapy, a process by which radiation plans are modified based on changes in tumor volume during treatment also enables dose escalation and minimization of normal tissue irradiation (Guckenberger et al., 2011).

Stereotactic radiosurgery (SRS) and SBRT are techniques for delivering very high doses in a single or in a few fractions (hypofractionation) in order to shorten treatment duration and escalate the biological equivalent dose (Dworzecki et al., 2012). SRS and SBRT require a high degree of precision requiring tumor motion compensation and improved targeting of metabolically active tumor. Radiation Therapy Oncology Group (RTOG) recently completed a multi-institutional North American phase II trial (RTOG 0236) using $54 \mathrm{~Gy}$ in three fractions SBRT for T1T2N0M0 NSCLC (Timmerman et al., 2010). Of the 55 evaluable patients, the 3-year primary tumor control rate was $97.6 \%$. The median overall survival in the cohort was 48.1 months. There were only nine patients that had grade III or higher treatment-related 
toxicity, and there were no deaths due to treatment. These results are better than historical data using conventionally fractionated radiotherapy for early-stage NSCLC.

The above-mentioned approaches to radiation therapy for lung tumors benefit from an accurate delineation of metabolically active primary tumor using PET/CT, which enables suitable dose escalation strategies to highly metabolic tumor volumes (MTVs).

\section{STAGING WITH F18 FDG PET/CT FOR THERAPY DECISION}

Accurate staging of NSCLC is essential for appropriate therapy selection. F18 FDG PET/CT shows higher accuracy compared to CT for mediastinal and distant metastases and affects management for approximately one-third of patients (Kernstine et al., 2002). Increased FDG uptake in metastatic nodes not significantly enlarged by CT criteria is the reason for superior sensitivity of PET/CT. PET/CT is also able to differentiate metabolically active lung tumor from atelectasis (Nestle et al., 1999; Figures 1-6) as well as intratumoral necrotic zones which helps better determine RT target volumes. It is therefore possible to escalate doses to viable tumor and minimizing radiation to normal tissue, which may reduce long-term fibrosis and related sequelae (De Ruysscher et al., 2005).

Delineation of mediastinal nodes by PET/CT is important for decision-making regarding surgery vs. radiation. NSCLC patients with no involved lymph nodes (N0) or only positive hilar nodes (N1) may have operable disease if the primary tumor is judged to be resectable. In non-operable patients who have T1 or T2 disease without nodal metastases (N0), SBRT delivering 6080 Gray in 6-8 fractions can potentially achieve excellent local control. Accurate detection of mediastinal lymph node metastases is critical for determining the application of SBRT for patients with early-stage NSCLC. Li et al. (2012) in a multi-center

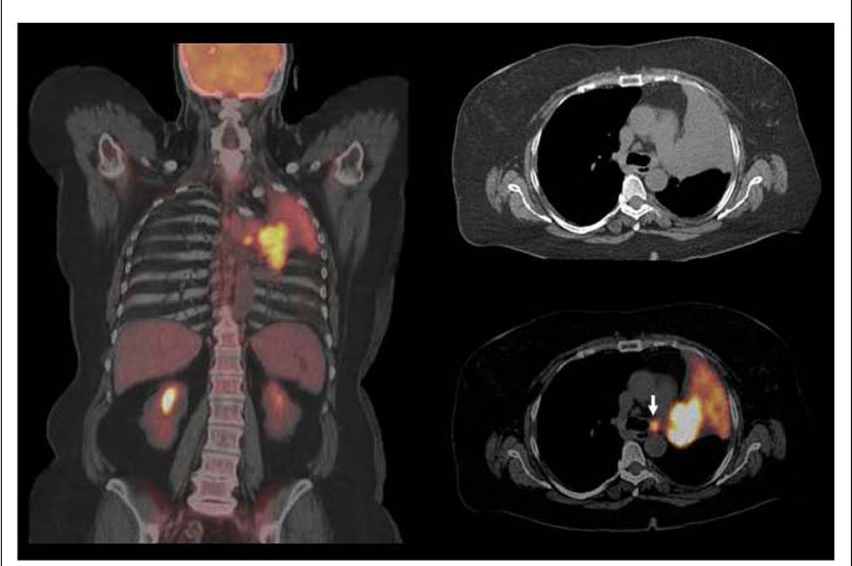

FIGURE 1 | Squamous cell carcinoma in left upper lobe with associated atelectasis. F18 FDG PET/CT shows large hypermetabolic suprahilar mass with a small FDG avid left paratracheal lymph node metastases. F18 FDG PET/CT defines the extent of metabolically active tumor and differentiates viable tumor from adjacent atelectasis. FDG avid solitary mediastinal lymph node metastases were also detected by PET/CT while CT was equivocal. However, the major impact of PET/CT was for guiding GTV with incorporating metabolically active tumor and nodal metastases and excluding atelectatic lung tissue in order to deliver higher dose to the tumor and decrease radiation to normal lung.

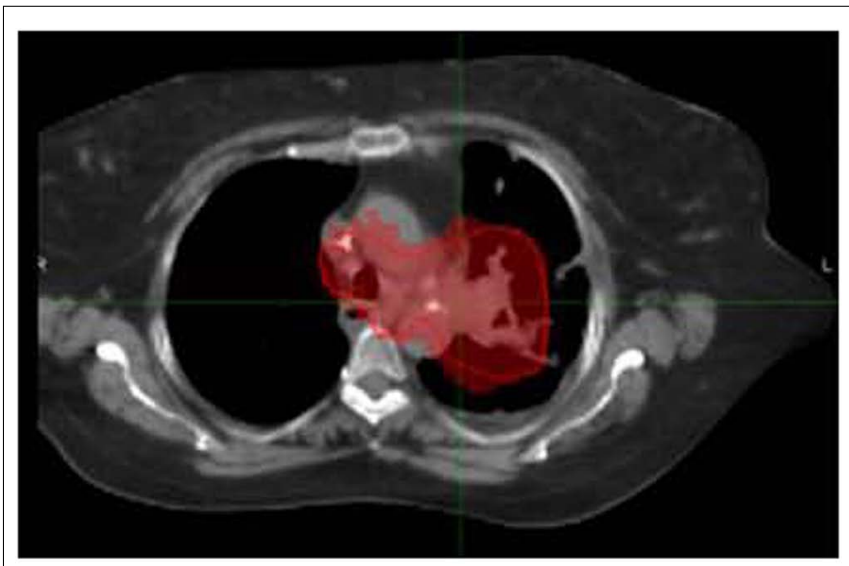

FIGURE 2 | The same patient in Figure 1A was treated with concurrent chemoradiation with a total dose of $66 \mathrm{~Gy}$ in $2 \mathrm{~Gy}$ fractions.

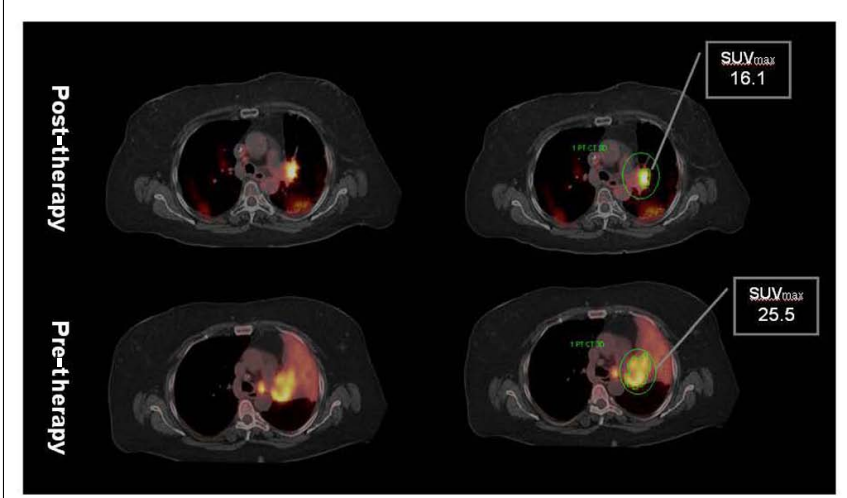

FIGURE 3 | Post-radiation therapy F18 FDG PET/CT shows significant decrease in tumor volume but as well as SUVmax. However,

considerable amount of metabolically active residual tumor is evident in post-therapy PET suggesting inadequate local control. Note absence of atelectasis with reinflation of the lung secondary to decrease in tumor volume. Minor post-radiation inflammatory changes in the posterior lung are characterized by mild increased FDG uptake. Data courtesy of M. D. Anderson, Cancer Center, Orlando, FL, USA.

study performed preoperative F18 FDG PET/CT in 200 patients. The PET findings related to lymph nodes were confirmed with histopathological examination of the surgical specimen. PET/CT demonstrated high specificity $(83 \%)$ and negative predictive value (NPV) (91\%) for presence of mediastinal lymph node metastases. The conclusion was that a negative PET/CT for mediastinal nodal metastases was sufficient evidence to justify treating the primary tumor with SBRT.

PET/CT can change the radiation field significantly by including FDG avid non-enlarged metastatic lymph nodes within the treatment field. While the sensitivity of CT for mediastinal node metastases detection is $56 \%$, for F18 FDG PET/CT, this is $83 \%$ for all stages, $91 \%$ when the CT scan shows enlarged lymph nodes, and 70\% for normal-sized lymph nodes (Hellwig et al., 2009). In view of the high NPV of F18 FDG PET/CT for nodal metastases $(>90 \%)$, routine elective nodal radiation is no longer recommended (Senan et al., 2004). It has been shown 


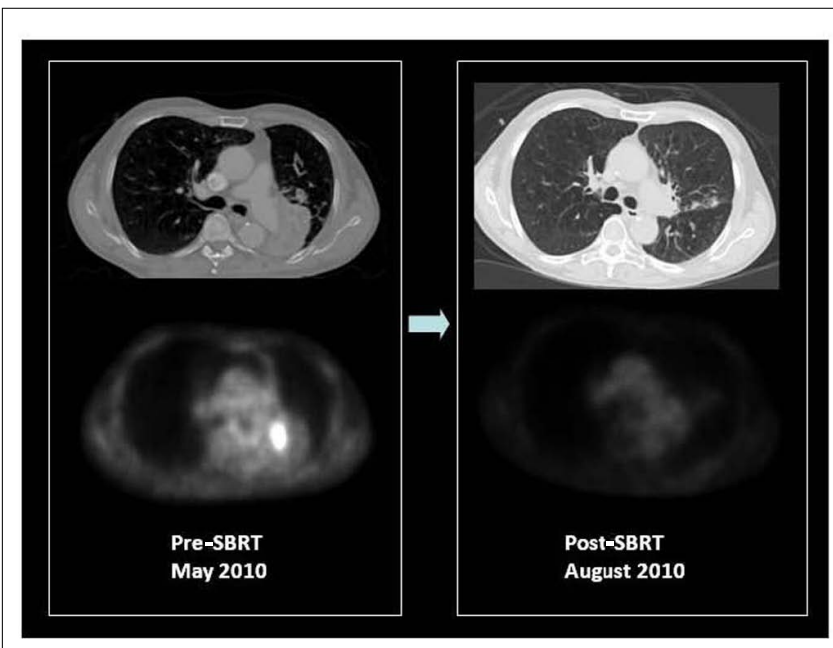

FIGURE 4 | F18 FDG PET/CT of a patient with medically inoperable, centrally located, stage I squamous cell carcinoma of the left lower lobe causing obstructive left lower lung collapse. Contrast CT is unable to differentiate tumor from adjacent collapsed left lower lobe. PET only image of the same axial slice demonstrates FDG avidity of only the tumor and differentiates metabolically active tumor from adjacent collapsed lung. PET was used for stereotactic body radiation therapy image-guided treatment planning to target the FDG avid tumor. Patient received $50 \mathrm{~Gy}$ in four fractions in May 2010. Early post-SBRT PET/CT imaging was performed in August 2010 to assess therapy response, which showed a complete metabolic response and re-inflation of the left lower lobe. The patient is currently free of local or distant recurrence or post-radiation complications with improved exercise tolerance and lowered oxygen consumption. Courtesy of UCLA Radiation Oncology SBRT program.

that irradiation of only PET positive lymph nodes is safe which reduces the high-dose volume (van Der Wel et al., 2005). Selective mediastinal lymph node irradiation based on PET with $18 \mathrm{~F}$ FDG yielded a low rate of treatment failure for isolated nodes, suggesting that reducing the target volume does not result in compromised local control (De Ruysscher et al., 2005). In a dosimetric study, van Der Wel et al. (2005) reported that for 21 patients with N2 or N3 NSCLC, the use of PET/CT in radiotherapy planning resulted in a lower level of radiation exposure of the esophagus and the lungs, allowing a significant increase in the dose delivered to the tumor. This is to a large extent due to avoidance of prophylactic radiation to mediastinal nodes if they are PET/CT negative. Similarly, dose escalation in PET positive areas with omitting of elective nodal irradiation in small-cell lung cancer was studied by Shirvani et al. (2010). Sixty-two patients were treated with IMRT based on PET/CT. The RT plan did not include elective nodal stations if they were PET negative. Only one patient out of 62 had recurrence in unirradiated elective nodes. Planning the target volume based on PET, 45 Gy was delivered in 30 twice-daily fractions. Most recurrences were either distant or within the high-dose volume and not in the initially PET negative elective nodal regions. This suggests that PET/CT-based radiation therapy planning obviates elective nodal irradiation even in smallcell lung cancer. Although PET-defined mediastinal radiotherapy fields appear to be safe, because of a false-positive rate of approximately 30\%, ideally pathological confirmation of PET-positive mediastinal nodes should be obtained by mediastinoscopy or endoscopic ultrasound-guided fine needle aspiration (EUS-FNA). In squamous cell carcinoma, sensitivity for mediastinal metastases was similar between EUS-FNA and FDG PET/CT (97.9 vs. 96.3\%). However in adenocarcinoma, EUS-FNA shows higher sensitivity (94.6 vs. 77.8\%; Hwangbo et al., 2009), prompting the authors to suggest pathological staging in case of adenocarcinoma with negative PET.

F18 FDG PET/CT frequently up stages the overall disease by detecting unsuspected distant metastasis ( $>20 \%$ of pre-PET stage III), commonly in liver, adrenal, and bone as well as identifies patients with very advanced loco regional (occult stage IIBIIIB) disease (Mac Manus et al., 2001b). Thus, PET/CT staging is important in minimizing the rate of futile thoracotomies for patients with occult systemic disease. In a large prospective trial, $30 \%$ of patients who were candidates for high-dose radiotherapy on the basis of conventional staging received only palliative therapies after PET, because of unexpected distant metastasis $(20 \%)$ or very extensive intrathoracic disease (10\%; Mac Manus et al., 2001a).

PET/CT has significantly improved the accuracy of staging for NSCLC and thus improved oncologists' ability to give the appropriate treatment for the correct stage. A prospective study of 105 consecutive patients with NSCLC showed that the addition of PET changed management from curative to palliative intent in $26 \%$ of patients and changed overall management in 67\% (Kalff et al., 2001). In a large study of 153 patients with NSCLC, PET changed the stage of $33 \%$ of the patients and changed the target volumes for radiation in 25\% (Hicks et al., 2001). The interobserver as well as intraobserver variability was significantly reduced when the F18 FDG PET imaging was available for tumor volume delineation (Fox et al., 2005).

\section{GROSS TUMOR VOLUME DELINEATION WITH F18 PET/CT-OPTIMUM THRESHOLDING}

Gross Tumor Volume (GTV) delineation based on hypermetabolic tumor has shown to be significantly different from GTV drawn on CT only (Bradley et al., 2004). PET/CT-based planning offers the potential of dose escalation to the tumor regions with the highest FDG uptake in order to potentially achieve improved local control as well as reduced excessive dose to organs at risk such as the lung, heart, and esophagus. In several planning studies, it was shown that GTVs based on PET were smaller than that with CT, thus leading to improved dosimetry to the organs at risk (Møller et al., 2011). Hanna et al. (2010) performed a second PET/CT for RT planning in 28 patients with stage IA-IIB NSCLC who already had undergone a staging PET/CT to assess the effect of additional planning PET/CT for GTV definition compared to CT-based planning alone. There was a significant reduction in interobserver variation for GTV delineation with the use of a planning PET/CT. There was a mean increase in GTV size of $18.8 \%$ using PET/CT compared to CT only planning for patient treated with radiotherapy only. For patients who received neoadjuvant chemotherapy, the GTV size using PET/CT was lower by only 5.2\% (Figures 7 and 8).

Meng et al. (2012) correlated microscopic extension of tumor on histopathology with SUVmax and GTV in NSCLC and found that microscopic extension is larger in tumors with higher SUVmax. The study suggested that margins of 1.93, 3.90, 


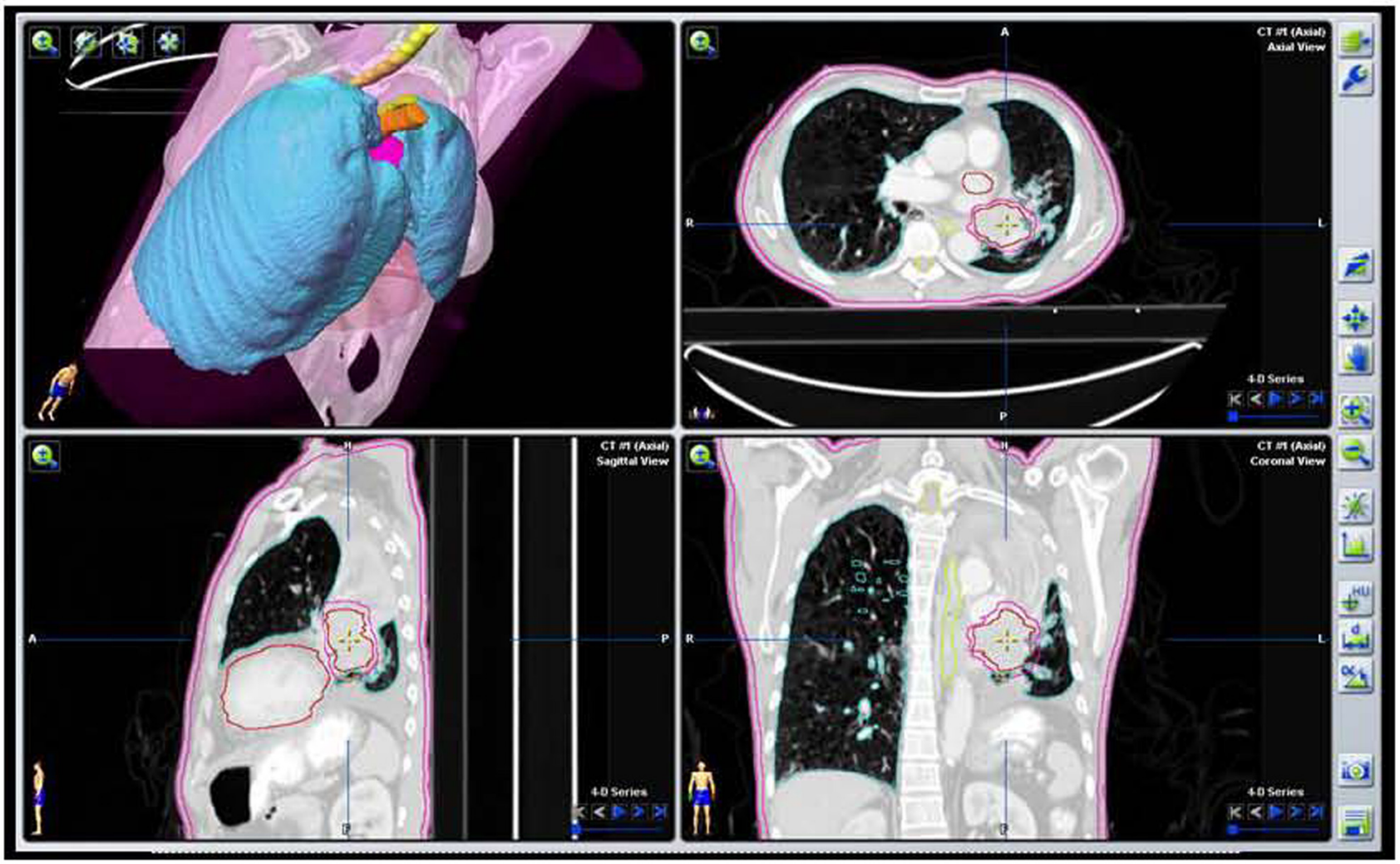

FIGURE 5 | Target volume contours of the same patient as Figure 2A based on the initial PET/CT data with VOI defining the GTV and PTV excluding collapsed lung tissue.

and $9.60 \mathrm{~mm}$ for SUVmax $\leq 5,5-10$, and $>10$ added to the GTV would be adequate to cover $95 \%$ of microscopic tumor extensions.

PET/CT-based contouring for radiation therapy planning was compared to CT-based planning in 50 lung cancer patients who underwent radiotherapy with a curative intent (Mac Manus, 2010). Radiation dose was planned for 60 Gy delivered in 30 fractions over 6 weeks. The study demonstrated that $37 \%$ patients would have had a grade I geographic miss and 26\% would have had a grade II geographic miss if radiotherapy planning was done without the use of PET imaging. A grade I geographic miss is defined as inadequate GTV coverage, while a grade II geographic miss is defined as inadequate coverage of PTV excluding the GTV.

The Radiation Therapy Oncology Group (RTOG) 0515 is a phase II prospective trial designed to quantify the impact of PET/CT compared with CT alone on radiation treatment plans (RTPs) in NSCLC (Bradley etal., 2012). Forty-seven patients underwent FDG PET/CT followed by definite RT (>60 Gy) based on PET/CT- generated radiation plan. Mean follow-up was for 12.9 months. GTVs derived from PET/CT were significantly smaller than that from CT only (mean GTV volume 86.2 vs. $98.7 \mathrm{ml}$ ). PET/CT changed nodal GTV contours in $51 \%$ of the patients. The elective nodal failure rate for GTVs derived by PET/CT was very low, supporting the RTOG standard of limiting the target volume to the primary tumor and involved nodes. Not surprisingly, mean lung dose using PET/CT-based planning was slightly lower than that with CT planning alone. However, there was no difference in the mean esophageal dose.

Although a fixed threshold to the highest standardized uptake value (SUV) for generating target volumes on PET has been used extensively, studies suggest that an adaptive thresholding approach is more suitable. Biehl et al. (2006) compared PETbased VOI using varying threshold levels compared to CT-based target volumes in 20 peripheral lung tumors with volumes in which the target volume is easily identified on the CT. The optimal PET threshold for the best match was tumor size-dependent; it was $15 \%$ of SUVmax for tumors larger than $5 \mathrm{~cm}$ and $42 \%$ of SUVmax for tumors less than $3 \mathrm{~cm}$. Thus adaptive thresholding based on tumor size and SUVmax may result in a more customized manner of defining the GTV. However, this is an area of controversy and active research. The optimal threshold for SUV for the specific use of target definition is still unknown.

\section{DOSE ESCALATION BASED ON SUV THRESHOLDS}

Local failure rates are high in NSCLC following radiation therapy, up to $50 \%$. If PET-based parameters such as SUV can predict sub-volume of the GTV at the highest risk for local recurrence after radiotherapy, than this information can be used for 


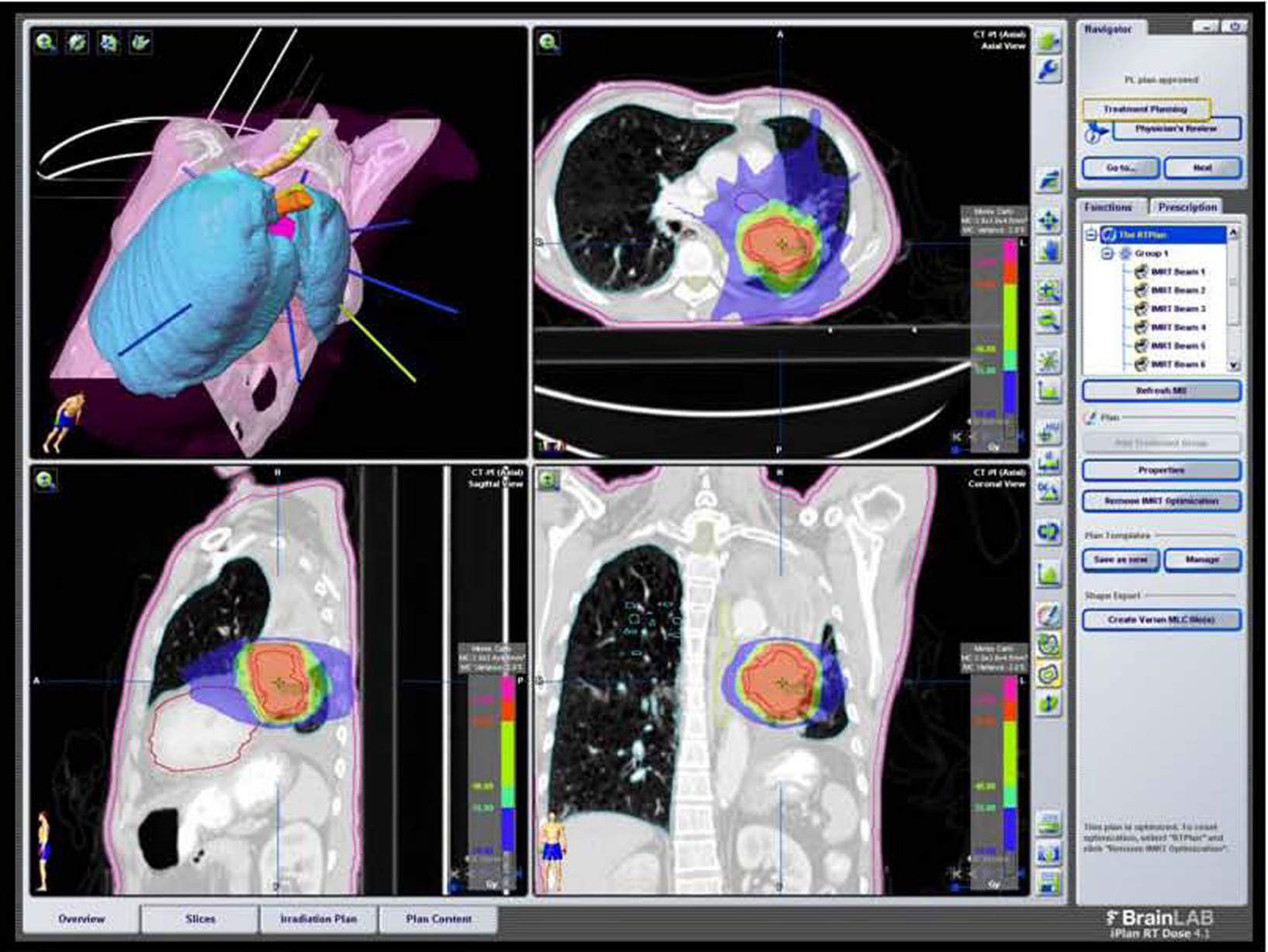

FIGURE 6 | SBRT dose wash for the same patient as Figure 2A with dose color wash [highest dose (50 Gy) in red delivered to the PTV]. Data courtesy of UCLA Radiation Oncology.

dose-intensifying the radio-resistant sub-volumes. Along this line, it has been shown that areas with the highest FDG uptake in the pre-therapy scans are the sites with the highest risk for local failure, suggesting escalating the dose to this sub-volume may improve local control. Aertis et al. (2009) performed pre and post F18 FDG PET/CT in 55 inoperable stage I-III NSCLC patients. Patients with residual metabolic-active areas within the tumors had a significantly worse survival compared to individuals with a complete metabolic response (CMR). The residual metabolic-active areas within the tumor largely corresponded with the volume that has a threshold of $50 \%$ of the SUVmax in the pre-radiotherapy scan. Thus a boost to a sub-volume encompassing the 50\% SUVmax sub-volume is a reasonable strategy to improve local control.

In another similar study by Abramyuk et al. (2009), 10 patients with locoregional relapse of NSCLC underwent F18 FDG PET/CT before, during, and in the 4-12 months following curative chemoradiotherapy (dose 66 Gy). CT showed partial reduction of tumor volume after radiotherapy in all patients. PET/CT revealed partial response in eight patients and CMR in two patients during RT. Six to nine months after RT all patients had a local failure.
Tumor recurrences were localized mostly in the areas with highest metabolic activity in the pre therapy PET/CT. This suggests that these areas with high metabolic activity are potentially the most radio-resistant. Thus, another strategy is to dose intensify areas of the highest metabolic activity based on the pre-treatment FDG $\mathrm{PET} / \mathrm{CT}$.

Feng et al. (2009) performed F18 FDG PET/CT in 14 patients with stage I-III NSCLC before RT and in mid-RT (after 40-50 Gy). 3D conformal RT plans were generated for each patient, first using only pre-treatment CT scans. Mid-RT PET volumes were then used to design boost fields. Mid-RT PET scan-based modification of radiation therapy plans allowed meaningful dose escalation of 30-102 Gy (mean 58 Gy) or decrease in the normal tissue complication probability in 10 of the 14 patients studied. There was a mean decrease in tumor volume after $40-50$ Gy of $26 \%$ on CT and $44 \%$ by PET/CT. The study concluded that there is a significant change in the tumor metabolic activity as well as the metabolic volume after 40-50 Gy and adaptation of RT based on the midtreatment PET/CT can help to escalate dose to active tumor as well as reduce dose to critical organs. 


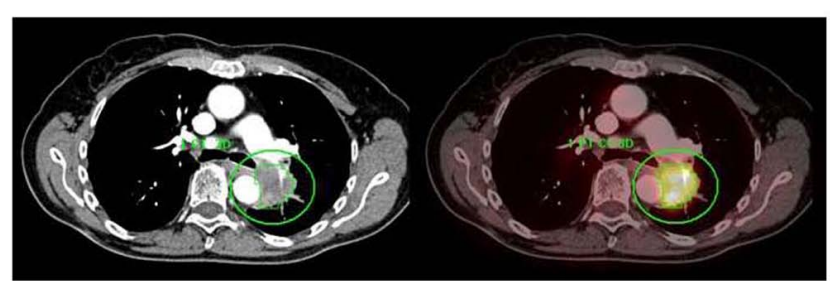

FIGURE 7 | A centrally located left upper lobe tumor. F18 FDG PET/CT shows large FDG avid mass without mediastinal metastases. Integrated contrast CT defines the relationship of tumor with adjacent aorta and pulmonary vessels. Automated 3D contouring of metabolically avid tumor was performed using a threshold of $40 \%$ of SUVmax. Volume of interest was exported as RT structure set for radiation planning.

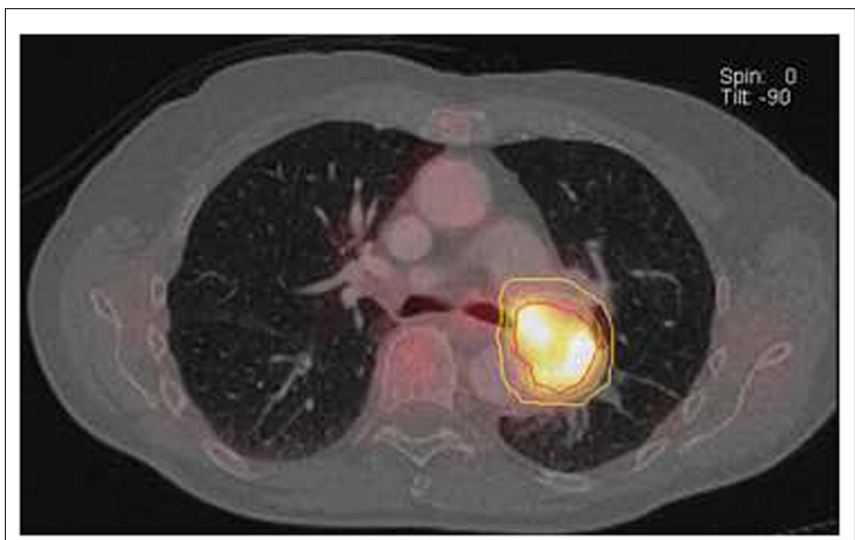

FIGURE 8 | GTV (red line) drawn based on $\mathbf{4 0} \%$ SUVmax threshold using an automated volume of interest (VOI) definition of

metabolically tumor. Clinical target volume (green line) and planning target volume (yellow line) generated by adding $0.5 \mathrm{~cm}$ and $1 \mathrm{~cm}$ margin to the GTV. Data courtesy of Karolinska Institute Sweden.

van Der Wel et al. (2005) compared CT and PET/CT-based planning in 21 NSCLC patients, with the goal to deliver $60 \mathrm{~Gy}$ in 30 fractions. F18 FDG PET/CT-based PTV was associated with reduced dose to the esophagus and normal lung and allowed significant radiation dose escalation while respecting all relevant normal tissue constraints. Based on FDG PET/CT planning, the mean esophageal V (45 Gy) decreased from 45.2 to $34.0 \%$ and the mean esophageal dose from 29.8 to 23.7 Gy. Similarly, average lung V (Hicks et al., 2001) decreased from 24.9 to $22.3 \%$ with slight decrease of the mean lung dose. For the same dose delivered to the lung, esophagus, and spinal cord, the mean dose to the target volume could be increased from $56 \mathrm{~Gy}$ with CT-based planning to 71 Gy with FDG PET/CT-based planning.

\section{RELATIONSHIP OF METABOLIC TUMOR VOLUME AND TUMOR PROGRESSION}

Stage at presentation is the primary prognostic factor in NSCLC. Tumor metabolic activity, as estimated by the SUV, is a prognostic factor in patients with NSCLC. MTV or the volume of tumor tissue demonstrating increased FDG uptake on PET, including primary tumor as well as metastases, is another independent adverse prognostic factor. Lee et al. (2012) evaluated 61 primary NSCLC patients (39 treated with curative intent while 22 treated with palliative intent) with F18 FDG PET/CT before and after therapy. MTV was found to be an independent prognostic factor after accounting for all traditional prognostic variables (stage, performance status, weight loss, etc.). In the subset of patients treated with a curative intent, i.e., stage I-III patients, higher MTV was significantly associated with worse survival and associated with a trend toward worse progression-free survival. Patients with MTV below the median for the respective therapy group showed higher PFS. The 2-year PFS for patients with MTV below median was 60 vs. $39.7 \%$ for those with higher MTV (median PFS 34.9 vs. 11.9 months).

\section{GATED PET/CT-BASED PLANNING}

Respiratory motion of lung tumors is a key factor which impacts radiation dose delivery to the tumor as well as irradiation of normal lung tissue. Respiratory motion can be as large as $3 \mathrm{~cm}$ for lesions in lung bases. One study (Hamill et al., 2008) reported that the range of tumor motion varied from 8 to $25 \mathrm{~mm}$ among five lung cancer patients. Respiratory motion assessment can also improve intratherapy modification of treatment plan to adapt to changes in tumor volume and motion during the treatment course.

Accurate estimation of motion and incorporation of such information into treatment planning is essential for SBRT as well as conventionally fractionated treatments with dose escalation. 4D CT-based planning has been widely used to account for motion in order to achieve smaller margins and reduce dose to normal tissue. In a study involving 10 NSCLC patient treated with SBRT (Wang et al., 2009), 4D CT-based planning provided the smallest treatment volume, permitted higher doses per fraction, and reduced doses to the normal lung compared to standard CT-based planning. However, studies have shown that fast CT imaging of a moving tumor can result in poor representation of the time averaged position and shape of the tumor. In a phantom-based study (Caldwell et al., 2003) PET imaging provided a more accurate representation of $3 \mathrm{D}$ volume of phantom tumors compared to gated CT.

Respiratory-correlated PET ameliorates motion blurring and enables visualization of lung tumor functional uptake throughout the breathing cycle but has achieved limited clinical use in radiotherapy planning. Tumor volumes generated by 4D CT and respiratory-gated PET were compared by Lamb et al. (2011) in four lower lobe lung tumors (4-18 cc in volume) in three patients. GTVs incorporating tumor motion were generated by combining tumor contours from multiple PET respiratory phase data as well as ungated PET images using standard tumor thresholding approach. GTV using CT was generated by combining end inspiration and end expiratory phases of 4D CT. The GTV volumes generated by gated PET were on an average 30\% lower than that generated by $4 \mathrm{D}$ CT. Volumes generated by ungated PET was also lower than that of 4D CT but the difference was smaller. Gated PET is particularly useful in lower lobe tumors with significant motion for SBRT planning and allows for more accurate representation of tumor motion than a $4 \mathrm{D}$ CT. $4 \mathrm{D}$ CT is often inaccurate for lesions in the lower lobe due to tumor proximity to soft tissue like the liver on the right side and stomach/spleen on the left side with the attendant decrease in the accuracy of tumor motion assessment. 
Bosmans et al. (2006) assessed 23 patients with locally advanced NSCLC who underwent F18 FDG PET/CT as well as respiratorygated CT prior to treatment, and repeated at the first and second weeks after the start of radiation. The changes in tumor motion during radiation therapy were relatively small. However, there was a great deal of variation in tumor size during therapy, ranging from $30 \%$ increase to similar decrease in tumor volume. The authors concluded that such changes in tumor size required replanning preferably with evaluation of tumor motion during the course of therapy (Figures 9 and 10).

\section{PET/CT-BASED RADIATION THERAPY FOLLOW-UP AND RECURRENCE DETECTION}

The SUVmax of primary NSCLC has been shown to be of prognostic value for survival outcomes. Correlation of pre- and post-RT SUVmax to overall survival as well as disease free survival shows

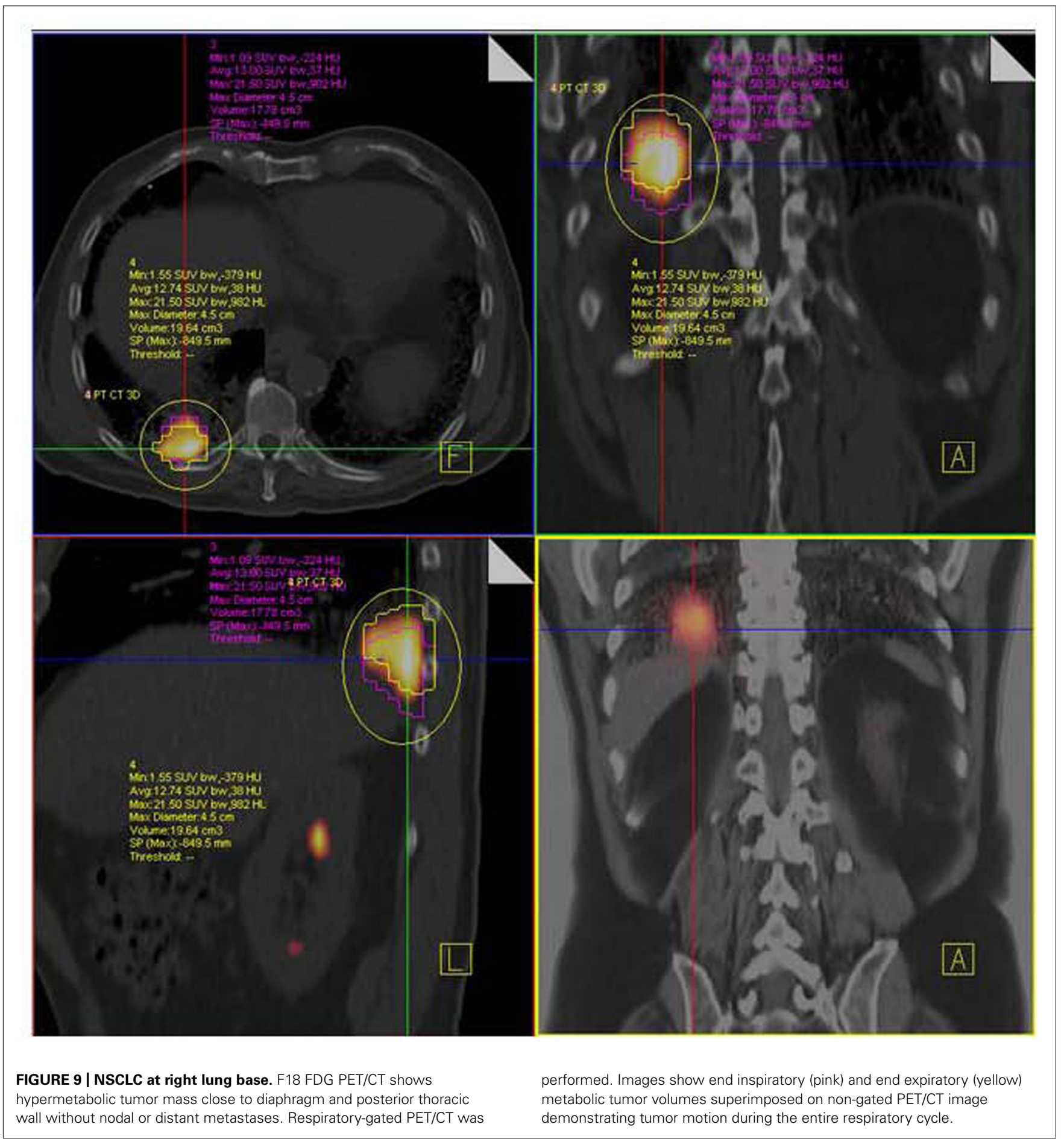




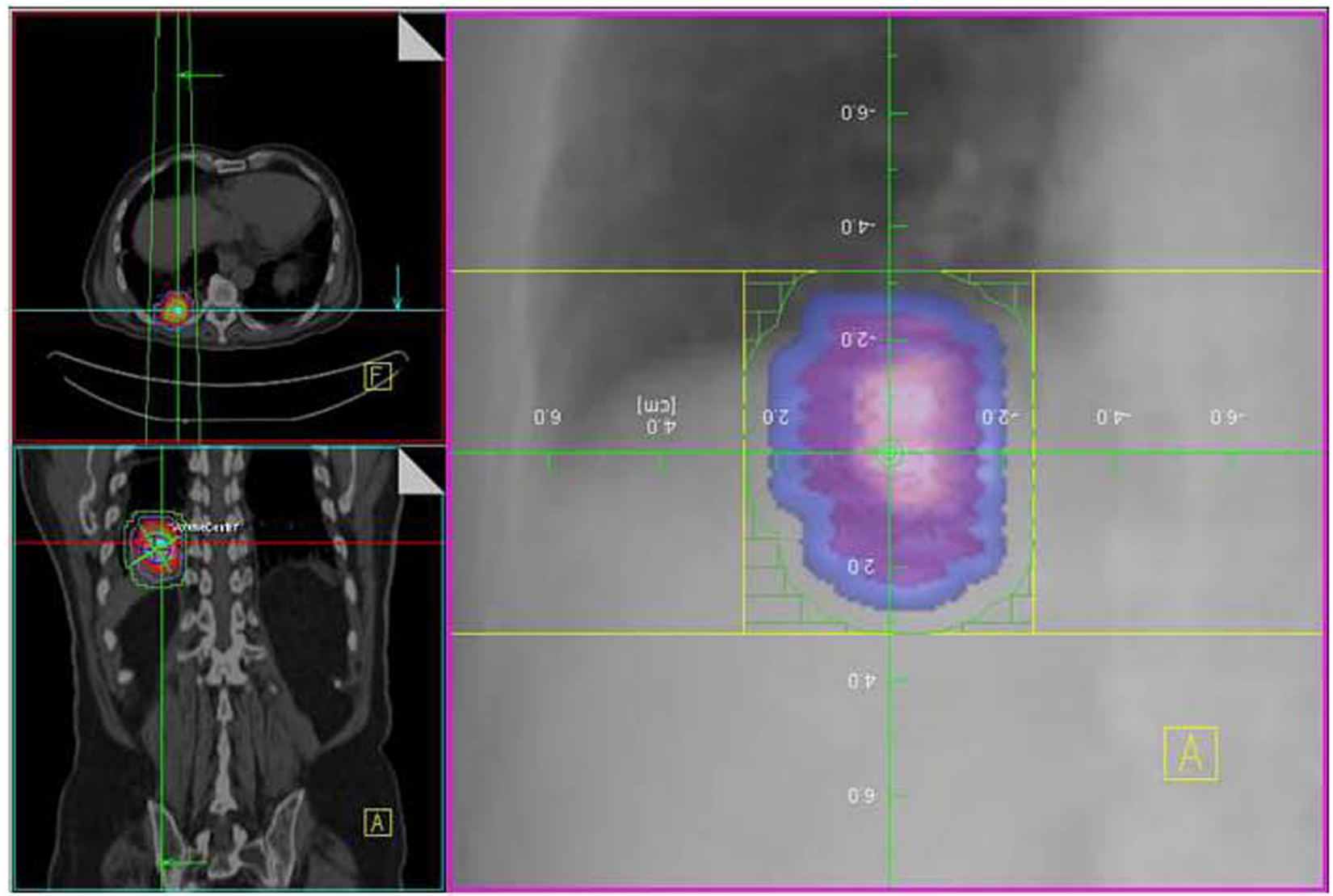

FIGURE 10 | Same patient as in Figure 4A with GTV (pink), CTV (blue), and PTV (green) drawn using a combination of end expiratory and end inspiratory metabolically active tumor volume as obtained from respiratory-gated PET/CT.

that higher SUVmax in primary tumor and nodal metastases was associated with poor survival (Liao et al., 2012). This is related to the fact that tumor glucose uptake may correlate with the metastatic potential.

The value of FDG PET in evaluation of response to RT has been established. In a series of 88 NSCLC patients (Mac Manus et al., 2005) who underwent pre- and post-chemo/RT (median 70 days after initiation of RT of $60 \mathrm{~Gy}$ in 30 fractions over 6 weeks), 45\% had CMR and 36\% PMR (partial metabolic response) on FDG PET. Median survival for CMR and non-CMR patients was 31 and 11 months, respectively. One-year survival for CMR and non-CMR patients was 93 and 47\%, respectively. This demonstrates the impact of CMR on follow-up PET after RT as a predictive marker of outcomes despite confounding issues such as radiotherapy-induced inflammation.

SUVmax changes during radiotherapy are significantly different between metabolic responders and non-responders. Metabolic responders have a better overall survival than the non-responders. Percentage decrease in SUVmax following RT directly correlates with disease-free survival (Lopez Guerra et al., 2012). Metabolic non-responders have been demonstrated to have a higher SUVmax at all time points investigated and showed a significant increase in SUVmax during the first week of irradiation followed by a decrease. In contrast, the responders showed a stable SUVmax during irradiation. The increase of SUVmax during the first week of RT was probably due to inflammation, since a rather high median dose of 19.8 Gy (1.8 Gy BID) was already delivered at the time point of the first repeat PET/CT scan.

FDG PET/CT-based response evaluation for lung tumors undergoing radiation therapy is often complicated due to postradiation inflammation. Hicks et al. (2004) assessed the relationship of post-radiation inflammatory changes in irradiated normal lung tissue and metabolic response in tumor in 73 patients with NSCLC. Metabolic response of tumor was greater in patients with higher inflammation-related FDG uptake in normal lung suggesting that tumor radio-responsiveness and normal tissue radiosensitivity may be linked. Inflammatory changes did not compromise evaluation of prognostic stratification by PET as long as a meticulous visual response assessment criterion was used.

$\mathrm{PET}$ also plays a role in detecting recurrent disease. Scarring and post-operative changes complicate interpretation of CT images and needle biopsy results are inconclusive in up to $30 \%$ patients (Mesurolle et al., 2003). Metabolic imaging with PET/CT however shows a high accuracy (between 78 and 98\%) in post-therapy NSCLC for recurrence detection (Erasums and Patz, 1999). Because post-radiation pneumonitis and inflammation are more 
common immediately after treatment, increasing the interval between the end of radiation therapy and the PET scan provides a greater specificity for abnormal findings. Earliest recommended interval is 6 weeks while $4-6$ months interval is common practice.

Metabolic response assessment is often used as a surrogate of local failure and survival. Early identification of patients with residual metabolic activity is essential as this enables selection of patients who could potentially benefit from additional therapy. Velasquez et al. (2010) performed pre and post radical chemo/RT PET/CT in 101 patients with inoperable NSCLC and found significantly reduced overall survival in patients with residual metabolic active areas in post therapy PET. Large tumors and tumors with higher SUVmax were more likely to be associated with residual metabolically active tumor at end of RT.

Sequential PET/CT imaging during radiation therapy has been shown to be beneficial for accurate evaluation of therapy response despite the issues related to post-radiation inflammation. EdetSanson et al. (2012) performed FDG PET/CT before and during radiation therapy (60-70 Gy, 2 Gy per fraction, 5 fractions per week) in 10 NSCLC patients. PET/CT was performed every seven fractions (14 Gy total dose increments) with all patients undergoing 5-6 PET/CT studies. All 17 lesions (6 tumors and 11 nodal metastases) showed progressive decrease in SUVmax with increase in cumulative radiation dose. A 50\% decrease in SUV max was obtained around a total dose of 45-50 Gy (during week 5 of RT).

Because of the increased FDG uptake in inflammation secondary to radiation therapy in lung cancer that can complicate PET/CT evaluation of response to radiation therapy, FLT, a proliferation agent has been advocated for RT follow-up. FLT is felt to have a lower level of uptake in inflammation compared to proliferating tumor cells. A pilot study (Everitt et al., 2009) performed baseline FDG and FLT PET/CT studies as well as follow-up FLT

\section{REFERENCES}

Abramyuk, A., Tokalov, S., Zophel, K., Koch, A., Szluha Lazanyi, K., Gillham, C., Herrmann, T., and Abolmaali, N. (2009). Is pre-therapeutical FDG-PET/CT capable to detect high risk tumor subvolumes responsible for local failure in non-small cell lung cancer? Radiother. Oncol. 91, 399-404.

Aertis, H. J., van Baardwijk, A. A., Petit, S. F., Offermann, C., Loon, J., Houben, R., Dingemans, A. M., Wanders, R., Boersma, L., Borger, J., Bootsma, G., Geraedts, W., Pitz, C., Simons, J., Wouters, B. G., Oellers, M., Lambin, P., Bosmans, G., Dekker, A. L., and De Ruysscher, D. (2009). Identification of residual metabolicactive areas within individual NSCLC tumours using a pre-radiotherapy (18)Fluorodeoxyglucose-PET-CT scan. Radiother. Oncol. 91, 386-392.

Biehl, K. J., Kong, F. M., Dehdashti, F., Forster, K., Siegel, B. A., Brunetti, J., Purdy, J., Faria, S., Vu, T., Thorstad, W., and Choy, H. (2006). 18F-FDG PET definition of gross tumor volume for radiotherapy of non-small cell lung cancer. Is a single standardized uptake value of threshold approach appropriate? J. Nucl. Med. 47, 1808-1812.

Bosmans, G., van Baardwijk, A., Dekker, A., Ollers, M., Boersma, L., Minken, A., Lambin, P., and De Ruysscher, D. (2006). Intrapatient variability of tumor volume and tumor motion during conventionally fractionated radiotherapy for locally advanced non-small-cell lung cancer: a prospective clinical study. Int. J. Radiat. Oncol. Biol. Phys. 66, 748-753.

Bradley, J., Bae, K., Choi, N., Forster, K., Siegel, B. A., Brunetti, J., Purdy, J., Faria, S., Vu, T., Thorstad, W., and Choy, H. (2012). A phase II comparative study of gross tumor volume definition with or without PET/CT fusion in dosimetric planning for non-small-cell lung cancer (NSCLC): primary analysis of Radiation Therapy Oncology Group (RTOG) 0515. Int. J. Radiat. Oncol. Biol. Phys. 82, 435-441.

PET/CT studies on day 2,8,15, and 29 of radiation therapy for a multi-time point evaluation of RT response. Baseline FLT uptake corresponded to FDG avid regions although the uptake levels were lower with FLT. Sequential FLT studies during therapy demonstrated a progressive decrease of uptake with a mean decrease of $58 \%$ in SUV on day 29 compared to baseline. A marked reduction of (Mac Manus et al., 2001a) FLT uptake in irradiated bone marrow was observed for all cases. This reduction was observed even after only $2 \mathrm{~Gy}$, and all patients demonstrated a complete absence of proliferating marrow after $10 \mathrm{~Gy}$.

\section{CONCLUSION}

F18 FDG PET/CT-based radiation therapy planning for NSCLC is able to improve GTV definition and dose escalation by accurately defining mediastinal metastases as well as differentiate viable tumor from atelectasis and necrosis. PET/CT study prior to therapy provides valuable prognostic information from SUV, SUV max, MTV etc. that helps guide the intensity and targeting of the planned radiotherapy. PET/CT-based RT planning is able to achieve clinically meaningful dose escalation while limiting dose to organs at risk. Adaptive RT planning, which uses mid-therapy PET/CT for dose modifications, can achieve further dose escalation and improve tumor control probability. Sequential PET/CT imaging has also been shown to be valuable in the evaluation of tumor response to radiation and recurrence detection. Current research with new biomarkers such as proliferation and hypoxia biomarkers show promise by incorporating different types of metabolic information. This will be important for rationale radiation dose painting and assisting future patient-centric adaptive radiotherapy.

Data courtesy of M. D. Anderson, Cancer Center, Orlando, FL, USA.

Bradley, J., Thorstad, W. L., Mutic, S., Miller, T. R., Dehdashti, F., Siegel, B. A., Bosch, W., and Bertrand, R. J. (2004). Impact of FDG-PET on radiation therapy volume delineation in non-small-cell lung cancer. Int. J. Radiat. Oncol. Biol. Phys. 59, 78-86.

Caldwell, C. B., Mah, K., Skinner, M., and Danjoux, C. E. (2003). Can PET provide the $3 \mathrm{D}$ extent of tumor motion for individualized internal target volumes? A phantom study of the limitations of CT and the promise of PET. Int. J. Radiat. Oncol. Biol. Phys. 55, 1381-1393.

De Ruysscher, D., Wanders, S., Minken, A., Lumens, A., Schiffelers, J., Stultiens, C., Halders, S., Boersma, L., Baardwijk, A., Verschueren, T., Hochstenbag, M., Snoep, G. Wouters, B., Nijsten, S., Bentzen, S. M., Kroonenburgh, M., Ollers, M. and Lambin, P. (2005). Effects of radiotherapy planning with a dedicated combined PET-CT-simulator of patients with non-small cell lung cancer on dose limiting normal tissues and radiation dose-escalation: a planning study. Radiother. Oncol. 77, 5-10.

Dworzecki, T., Idasiak, A., Sygula, D., Dworzecka, U., and Suwiñski, R. (2012). Stereotactic radiotherapy as a sole or salvage therapy in non-small cell lung cancer patients. Neoplasma 59, 114-120.

Edet-Sanson, A., Dubray, B., Doyeux, K., Back, A., Hapdey, S., Modzelewski, R., Bohn, P., Gardin, I., and Vera, P. (2012). Serial assessment of FDG-PET FDG uptake and functional volume during radiothreapy (RT) in patients with non-small cell lung cancer (NSCLC). Radiother. Oncol. 102, 251-257.

Erasums, J, and Patz, E. Jr. (1999). Positron emission tomography imaging in the thorax. Clin. Chest Med. 20, 715-724.

Everitt, S., Hicks, R. J., Ball, D., Kron, T., Schneider-Kolsky, M., Walter, T., Binns, D., and Mac Manus, M. (2009). Imaging cellular proliferation during chemoradiotherapy: a pilot study of serial 
18F-FLT positron emission tomography/computed tomography imaging for non-small-cell lung cancer. Int. J. Radiat. Oncol. Biol. Phys. 75, 1098-1104.

Feng, M., Kong, F. M., Gross, M., Fernando, S., Hayman, J. A., and Ten Haken, R. K. (2009). Using flurodeoxyglucose positron emission tomography to assess tumor volume during radiotherapy for non-smallcell lung cancer and its potential impact on adaptive dose escalation and normal tissue sparing. Int. J. Radiat. Oncol. Biol. Phys. 73, 1228-1234.

Fox, J. L., Rengan, R., O'Meara, W., Yorke, E., Erdi, Y., Nehmeh, S., Leibel, S. A., and Rosenzweig, K. E. (2005). Does registration of PET and planning CT images decrease interobserver and intraobserver variation in delineating tumor volumes for nonsmall-cell lung cancer? Int. J. Radiat. Oncol. Biol. Phys. 62, 70-75.

Guckenberger, M., Richter, A., Wilbert, J., Flentje, M., and Partridge, M. (2011). Adaptive radiotherapy for locally advanced non-small-cell lung cancer does not underdose the microscopic disease and has the potential to increase tumor control. Int. J. Radiat. Oncol. Biol. Phys. 81, e275-e282.

Hamill, J. J., Bosmans, G., and Dekker, A. (2008). Respiratory gated CT as a tool for the simulation of breathing artifacts in PET and PET/CT. Med. Phys. 35, 576-585.

Hanna, G. G., McAleese, J., Carson, K. J., Stewart, D. P., Cosgrove, V. P., Eakin, R. L., Zatari, A., Lynch, T., Jarritt, P. H., Young, V. A., O’Sullivan, J. M., and Hounsell, A. R. (2010). (18)F-FDG PET-CT simulation for non-small cell lung cancer: effect in patients already staged by PET-CT. Int. J. Radiat. Oncol. Biol. Phys. 77, 24-30.

Hellwig, D., Baum, R. P., and Kirsch, C. (2009). FDG-PET, PET/CT and conventional nuclear medicine procedures in the evaluation of lung cancer: a systematic review. Nuklearmedizin 48, 59-69.

Hicks, R. J., Kalff, V., Mac Manus, M. P., Ware, R. E., Hogg, A., McKenzie, A. F., Matthews, J. P., and Ball, D. L. (2001). (18)F-FDG PET provides high impact and powerful prognostic stratification in staging newly diagnosed non-small-cell lung cancer. J. Nucl. Med. 42, 1596-1604.

Hicks, R. J., Mac Manus, M. P., Matthews, J. P., Hogg, A., Binns, D., Rischin, D., Ball, D. L., and Peters, L. J. (2004). Early FDG-PET imaging after radical radiotherapy for non-small-cell lung cancer: inflammatory changes in normal tissues correlated with tumor response and do not confound therapeutic response evaluation. Int. J. Radiat. Oncol. Biol. Phys. 60, 412-418.

Hwangbo, B., Kim, S. K., Lee, H.-S., Lee, H. S., Kim, M. S., Lee, J. M., Kim, H.-Y., Lee, G.-K., Nam, B.H., and Zo, J. I. (2009). Application of endobronchial ultrasound-guided transbronchial needle aspiration following integrated PET/CT in mediastinal staging of potentially operable non-small cell lung cancer. Chest 135 , 1280-1287.

Kalff, V., Hicks, R. J., Mac Manus, M. P, Binns, D. S., McKenzie, A. F., Ware, R. E., Hogg, A., and Ball, D. L. (2001). Clinical impact of (18)F fluorodeoxyglucose positron emission tomography in patients with nonsmall- cell lung cancer: a prospective study. J. Clin. Oncol. 19, 111-118.

Kernstine, K. H., Mclaughlin, K. A., Menda, Y., Rossi, N. P., Kahn, D. J., Bushnell, D. L., Graham, M. M., Brown, C. K., and Madsen, M. T. (2002). Can FDG-PET reduce the need for mediastinoscopy in potentially resectable non-small cell lung cancer? Ann. Thorac. Surg. 73, 394-401.

Lamb, J. M., Robinson, C., Bradley, J., Laforest, R., Dehdashti, F. White, B. M., Wuenschel, S., and Low, D. A. (2011). Generating lung tumor internal target volumes from 4D-PET maximum intensity projections. Med. Phys. 38, 5732-5737.

Lee, P., Bazan, J. G., Lavori, P. W., Weerasuriya, D. K., Quon, A., Le, Q. T., Wakelee, H. A., Graves, E. E., and Loo, B. W. (2012). Metabolic tumor volume is an independent prognostic factor in patients treated definitively for non-small-cell lung cancer. Clin. Lung Cancer 13, 52-58.

Li, X., Zhang, H., Xing, L., Ma, H., Xie, P., Zhang, L., Xu, X., Yue, J., Sun, X., Hu, X., Chen, M., Xu, W., Chen, L., and Yu, J. (2012). Mediastinal lymph nodes staging by 18F-FDG PET/CT for early stage non-small cell lung cancer: a multi center study. Radiother. Oncol. 102, 246-250.

Liao, C. T., Fan, K. H., Lin, C. Y., Yeh, J. J., and Kao, C. H. (2012). Meta-analysis study of lymph node staging by 18 F-FDG PET/CT scan in non-small cell lung cancer. Comparison of TB and non-TB endemic regions. Eur. J. Radiol. [Epub ahead of print].
Lopez Guerra, J. L., Gladish, G., Komaki, R., Gomez, D., Zhuang, Y., and Liao, Z. (2012). Large decreases in standardized uptake values after definitive radiation are associated with better survival of patients with locally advanced non-small cell lung cancer. J. Nucl. Med. 53, 225-233.

Mac Manus, M. P., Hicks, R. J., Ball, D. L., Kalff, V., Matthews, J. P., Salminen, E., Khaw, P., Wirth, A., Rischin, D., and McKenzie, A. (2001a). F-18 fluorodeoxyglucose positron emission tomography staging in radical radiotherapy candidates with nonsmall cell lung carcinoma: powerful correlation with survival and high impact on treatment. Cancer 92, 886-895.

Mac Manus, M. P., Hicks, R. J., Matthews, J. P., Hogg, A., McKenzie, A. F., Wirth, A., Ware, R. E. and Ball, D. L. (2001b). High rate of detection of unsuspected distant metastases by PET in apparent stage III non-small-cell lung cancer: implications for radical radiation therapy. Int. J. Radiat. Oncol. Biol. Phys. 50, 287-293.

Mac Manus, M. P. (2010). Use of PET/CT for staging and radiation therapy planning in patients with non-small cell lung cancer. $Q$. J. Nucl. Med. Mol. Imaging 54, 510-520.

Mac Manus, M. P., Hicks, R. J., Matthews, J. P., Wirth, A., Rischin, D., and Ball, D. L. (2005). Metabolic (FDG-PET) response after radical radiotherapy/chemoradiotherapy for non-small cell lung cancer correlates with pattern of failure. Lung Cancer 49, 95-108.

Meng, X., Sun, X., Mu, D., Xing, L., Ma, L., Zhang, B., Zhao, S., Yang, G. Kong, F. M., and Yu, J. (2012). Noninvasive evaluation of microscropic tumor extensions using standardized uptake value and metabolic tumor volume in non-small-cell lung cancer. Int. J. Radiat. Oncol. Biol. Phys. 82, 960-966.

Mesurolle, B., Mignon, F., Meingan, P., Domenge, C., Vasile, M., and Sigal, R. (2003). Head and neck cancer patients with pulmonary nodules: value and role of CT-guided transthoracic needle aspiration biopsies. Head Neck 25, 889-894.

Møller, D. S., Khalil, A. A., Knap, M. M., Muren, L. P., and Hoffmann, L. (2011). A planning study of radiotherapy dose escalation of PET-active tumor volumes in non-small cell lung cancer patients. Acta Oncol. 50, 883-888.
Nestle, U., Walter, K., Schmidt, S., Licht, N., Nieder, C., Motaref, B., Hellwig, D., Niewald, M., Ukena, D., Kirsch, C. M., Sybrecht, G. W., and Schnabel, K. (1999). 18F-Deoxyglucose positron emission tomography (FDG-PET) for the planning of radiotherapy in lung cancer: high impact in patients with atelectasis. Int. J. Radiat. Oncol. Biol. Phys. 44, 593-597.

Rubin, P. (2001). Clinical Oncology, 8th Edn. Philadelphia, PA: Saunders.

Senan, S., De Ruysscher, D., Giraud, P., Mirimanoff, R., Budach, V., and Radiotherapy Group of European Organization for Research and Treatment of Cancer. (2004). Literature-based recommendations for treatment planning and execution in high-dose radiotherapy for lung cancer. Radiother. Oncol. 71, 139-146.

Shirvani, S. M., Komaki, R., Stewart, D. J., Lippman, S. M., and Chang, J. Y. (2010). PET/CT-guided involvedfield intensity modulated radiation therapy for limited-stage small cell lung cancer. Int. J. Radiat. Oncol. Biol. Phys. 78, S34-S35.

Teh, B. S., Woo, S. Y., and Butler, E. B. (1999). Intensity modulated radiation therapy (IMRT): a new promising technology in radiation oncology. Oncologist 4, 433-442.

Timmerman, R., Paulus, R., Galvin, J., Michalski, J., Straube, W., Bradley, J., Fakiris, A., Bezjak, A., Videtic, G., Johnstone, D., Fowler, J., Gore, E., and Choy, H. (2010). Stereotactic body radiation therapy for inoperable early stage lung cancer. JAMA 303, 1070-1076.

van Der Wel, A., Nijsten, S., Hochstenbag, M., Lamers, R., Boersma, L., Wanders, R., Lutgens, L., Zimny, M., Bentzen, S. M., Wouters, B., Lambin, P., and De Ruysscher, D. (2005). Increased therapeutic ratio by 18FDG-PET-CT planning in patients with clinical CT stage N2/N3 M0 non-small cell lung cancer (NSCLC): a modeling study. Int. J. Radiat. Oncol. Biol. Phys. 61, 648-654.

Velasquez, E. R., Aerts, H. J., Oberije, C., De Ruysscher, D., and Lambin, P. (2010). Prediction of residual metabolic activity after treatment in NSCLC patients. Acta Oncol. 49, 1033-1039.

Wang, L., Hayes, S., Paskalev, K., Jin, L., Buyyounouski, M. K., Ma, C. C., and Feigenberg, S. (2009). Dosimetric comparison of stereotactic body radiotherapy using $4 \mathrm{D}$ CT and multiphase CT images for treatment planning of lung cancer: evaluation of the impact on daily dose coverage. Radiother. Oncol. 91, 314-324. 
Wurm, R. E., Gum, F., Erbel, S., Schlenger, L., Scheffler, D., Agaoglu, D., Schild, R., Gebauer, B., Rogalla, P., Plotkin, M., Ocran, K., and Budach, V. (2006). Image guided respiratory gated hypofractionated Stereotactic Body Radiation Therapy (H-SBRT) for liver and lung tumors: initial experience. Acta Oncol. 45, 881-889.
Conflict of Interest Statement: The author declares that the research was conducted in the absence of any commercial or financial relationships that could be construed as a potential conflict of interest.

Received: 26 April 2012; accepted: 25 June 2012; published online: 11 July 2012.
Citation: Lee P, Kupelian P, Czernin J and Ghosh P (2012) Current concepts in F18 FDG PET/CT-based radiation therapy planning for lung cancer. Front. Oncol. 2:71. doi: 10.3389/fonc.2012. 00071

This article was submitted to Frontiers in Cancer Imaging and Diagnosis, a specialty of Frontiers in Oncology.
Copyright $\odot 2012$ Lee, Kupelian, Czernin and Ghosh. This is an open-access article distributed under the terms of the Creative Commons Attribution License, which permits use, distribution and reproduction in other forums, provided the original authors and source are credited and subject to any copyright notices concerning any third-party graphics etc. 